\title{
Mathematical Model Applied to Monitoring the Glucose-Insulin Interaction inside the Body of Diabetes Patients
}

\author{
Sonia Akter ${ }^{\mathrm{a}}$, Md. Sirajul Islam ${ }^{\mathrm{a}}$, Md. Haider Ali Biswas ${ }^{\mathrm{b}, *}$ and Sajib Mandal ${ }^{\mathrm{a}}$ \\ ${ }^{a}$ Department of Mathematics, BSMRSTU, Gopalganj-8100, Bangladesh \\ ${ }^{b}$ Mathematics Discipline, Khulna University, Khulna-9208, Bangladesh
}

\begin{abstract}
The incidence and prevalence of diabetes are increasing all over the world and complication of diabetes constitutes a burden for the individuals and whole society. In this paper, we propose a mathematical model for monitoring glucose-insulin regulatory system in the human body. The non-linear cases are considered, and the model is analysed by using Lyapunov's method. The mathematical model, discussed the critical situation of the diabetes patients as well as for normal person are analysed for stability. The numerical approximation is used to verify the analytical results and the obtained solutions represent the complex situation of diabetes patients.
\end{abstract}

(C) 2020 Published by Bangladesh Mathematical Society

Received: 11 November, 2019 Accepted: 30 March, 2020

Keywords: Diabetes Mellitus; glucose-insulin regulatory system; mathematical model; Lyapunov's method; stability and numerical approximation.

AMS Subject Classification 2010: 92D30; 49K15; 34A34

\section{Introduction}

Diabetes mellitus, commonly known as diabetes, is a syndrome of disordered metabolism, usually due to a combination of hereditary and environmental causes, resulting in abnormally high blood sugar levels known as hyperglycaemia [1]. Glucose concentration in the blood of a normal person lies in the range of $80-110 \mathrm{mg} / \mathrm{dl}$

* Corresponding Author: Md. Haider Ali Biswas, E-mail address: mhabiswas@yahoo.com 
under fasting condition. Blood glucose levels are controlled by a complex interaction of multiple chemicals and under fasting condition. Blood glucose levels are controlled by a complex interaction of multiple chemicals and hormones in the body, including the hormones glucagon and insulin produced in the alpha and beta cells of the pancreas respectively. Diabetes has become an epidemic with considerable complications such as retinopathy, nephropathy, peripheral neuropathy and blindness. The number of diabetics in the world is increasing every year. More than 400 million people live with. Diabetes caused 5 million deaths in 2015; every six seconds a person dies from diabetes. More than 542,000 children have diabetes in 2015 and more than 20.9 million life births were affected by diabetes during pregnancy in 2015 [4]. In general, three forms of diabetes are considered: Type 1 diabetes, also known as Insulin Dependent Diabetes Mellitus (IDDM), affecting people under the age of 40 and representing 10-15\% of the diabetic population [3], Type 2 diabetes formerly known as Non-Insulin Dependent Diabetes Mellitus (NIDDM), representing the major part (85-90\%) [1] and Gestational diabetes (GDM) is a temporary condition that occurs in pregnancy and carries long-term risk of type 2 diabetes [1]. A detailed background and survey on diabetes mellitus is described in [1].

In recent times, mathematical modelling has become the most powerful tool to incorporate the dynamic behaviours of non-infectious diseases. Mathematical modelling is basically referred to as a method of simulating real-life situations with mathematical equations to forecast their future behaviour. Numerous mathematical models have been developed to identify the characteristics of glucose-insulin regulation inside the human body [2] [11] [12]. Many researchers have used mathematical models to understand and predict the behaviour of biological systems. The study of glucose-insulin interaction dates back as early as the six-ties and since then has been studied extensively by many researchers. The most widely used model in the study of diabetes is the minimal model which is used in the interpretation of the intravenous glucose tolerance test (IVGTT) [3]. Glucose-insulin regulation dynamic model, a set of ordinary differential equations (ODE) glucose-insulin interaction inside the human body, has been proven useful for understanding the pathogenesis of diabetes mellitus and developing treatment strategies. In this paper, we have shown the present scenario of diabetes mellitus in Bangladesh. Also we have studied a three-compartmental glucose-insulin regulation model and investigated their stability at disease free and endemic equilibrium points.

\section{Current Status of Diabetes Mellitus in Bangladesh}

Diabetes is a worldwide curse. There is no such country where this endemic disease does not exist. Although Bangladesh is still considered to be a high responded diabetes affected country in world, the present situation indicates that the influence of this endemic disease is gradually increasing.

Bangladesh has a disproportionately high diabetes population with more than 7.1 million, $8.4 \%$ or 10 million in 2015 [13], of the adult population affected by the diseases. The number will be 13.6 million in 2040. Nearly half of the population with diabetes, $51.2 \%$, don't know they have diabetes and don't receive any treatment. Bangladesh is home to a 161 million populations, according to the latest census report. During 90s, the country has a relatively low diabetes affected population. In 1995 is was only 4\% which is grew to 5\% in 2000 and $9 \%$ in 2006 to 2010. Here we show a graphical representation of diabetes mellitus surveillance of Bangladesh (see Figure 2.1) from 1980 to 2014 [13]. 


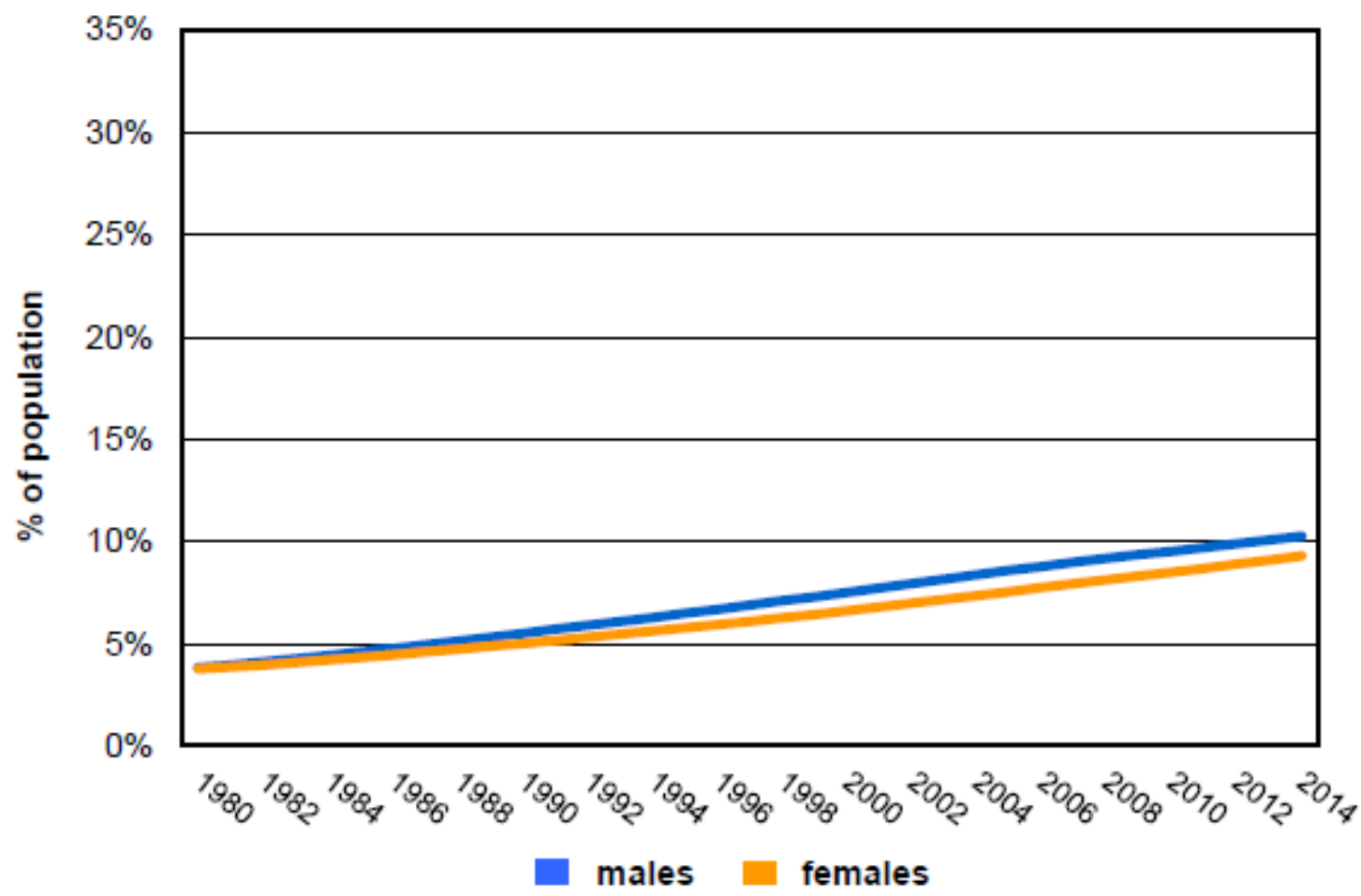

Figure 2.1: Trends in age-standardized (30-69 years) prevalence of diabetes in Bangladesh.

\section{Three Compartmental Glucose-Insulin Regulation Model}

Mathematical models have provided one mean of understanding diabetes dynamics. In this paper, we present a three compartmental model of glucose-insulin regulation inside the human body. In this model, we use three differential equations the first equation describing the glucose clearance, second equation describing the glucose uptake activity and third equation describing the insulin kinetics inside the human body, In this three compartmental model, let $G(t)$ is the plasma glucose concentration at time $t, X(t)$ is the generalized insulin variable for the remote compartment, $I(t)$ is the plasma insulin concentration at time $t$. The total population at $t$ time is represented by $N(t)=G(t)+X(t)+I(t)$.To describe the disease transmission in a certain population, $G_{b}$ is the basal blood glucose concentration, $I_{b}$ is the basal blood insulin concentration, $a_{1}$ is the glucose clearance rate independent of insulin, $a_{2}$ is the rate of clearance of active insulin, $a_{3}$ is the insulin independent increase in glucose uptake ability in tissue per unit of insulin concentration $I_{b}, a_{4}$ is the rate of the pancreatic $\beta$-cells' release of insulin after the glucose injection and with glucose concentration $G_{b}, a_{5}$ is the first order decay rate for insulin in plasma pancreatic $\beta$-cells release insulin. The schematic diagram of the model is shown in Figure 3.1. 


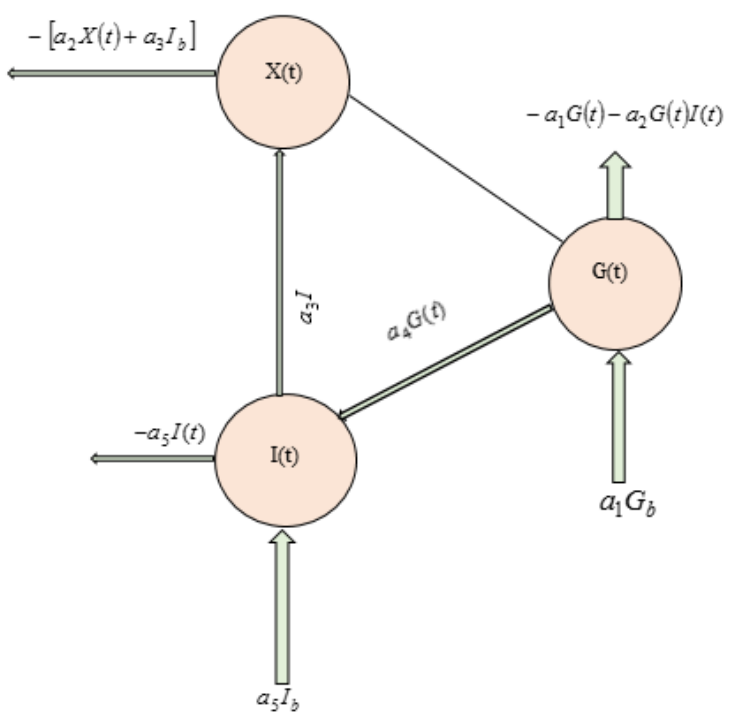

Figure 3.1: Schematic diagram of the glucose-insulin regulation model.

Taking the diagram in Figure 3.1 into account, our improved model [14] is governed by the following ordinary differential equations:

$$
\begin{array}{ll}
\frac{d G}{d t}=-a_{1} G-a_{2} G I+a_{1} G_{b} ; & G(0)=I_{0} \\
\frac{d X}{d t}=-a_{2} X+a_{3} I-a_{3} I_{b} ; & X(0)=X_{0} \\
\frac{d I}{d t}=a_{4} G-a_{5} I+a_{5} I_{b} ; & I(0)=I_{0}
\end{array}
$$

\section{Mathematical Analysis of Model}

Here we investigate the positivity of the model, find out different equilibrium points and check the stability using Lyapunov's method.

\subsection{Positivity of the Solution}

Here we check the positivity of each compartments such as glucose clearance $G$, glucose uptake activity $X$ and insulin kinetics $I$. We must have the positive values of these biological compartments. To test the positivity of these biological compartments, we need the following Lemma 4.1.

Lemma 4.1: Let $G(0)>0, X(0) \geq 0$ and $G(0)>0, X(0) \geq 0, I(0) \geq 0 \in \psi$, then the solutions $G(t), X(t), I(t)$ of the model system of equations (3.1) is positive. 


\section{Proof:}

To prove the Lemma 4.1, we have used the system of equations of the model (3.1),

$\frac{d G}{d t}=-a_{1} G-a_{2} G I+a_{1} G_{b}$

in order to find the positivity, we have,

$\frac{d G}{d t} \geq-a_{1} G+a_{1} G_{b}$

$\frac{d G(t)}{d t}+\mathrm{a}_{1} \mathrm{G}(\mathrm{t}) \geq \mathrm{p}_{1} \mathrm{G}_{\mathrm{b}}$

$\therefore \mathrm{IF}=e^{\int a_{1} d t} \geq e^{a_{1} t}$

Multiplying on both sides of (4.1) by $e^{a_{1} t}$, we have,

$e^{a_{1} t} \frac{d G(t)}{d t}+\mathrm{a}_{1} e^{a_{1} t} \mathrm{G}(\mathrm{t}) \geq \mathrm{a}_{1} \mathrm{G}_{\mathrm{b}} e^{a_{1} t}$

$\frac{d}{d t}\left(G e^{a_{1} t}\right) \geq a_{1} G_{b} e^{a_{1} t}$

$d\left(G e^{a_{1} t}\right) \geq d t\left(A e^{a_{1} t}\right)$

where, $A=a_{1} G_{b}$.

Now integrating (4.2), we have,

$G e^{a_{1} t} \geq e^{a_{1} t} \frac{A}{a_{1}}+C_{1}$

where $C_{1}$ is constant. Applying the initial condition at $t=0, G(t) \geq G(0)$. Hence from (4.3), we have,

$G \geq \frac{A}{a_{1}}+C_{1}$

$C_{1} \geq\left(\frac{A}{a_{1}}-G\right)$

Putting the value of $C_{1}$ into (4.3), we have,

$G e^{a_{1} t} \geq e^{a_{1} t} \frac{A}{a_{1}}+\left(\frac{A}{a_{1}}-G\right)$

$G(t) \geq \frac{A}{a_{1}}+\left(G-\frac{A}{a_{1}}\right) e^{-a_{1} t}$

Hence $G(t)>0$ at $t=0$ and $t \rightarrow \infty$. Similarly we can find the positivity of $X(t)$ and $I(t)$ under the initial conditions.

Therefore, it is true that, $(G(0)>0, X(0) \geq 0, I(0) \geq 0, \forall t \geq 0)$.

Lemma 4.2: The set $\psi=\left\{(G, X, I): 0 \leq G+X+I \leq c e^{-\delta t}+M, \delta=\min \left\{\left(a_{1}-a_{4}\right), a_{2},\left(a_{5}-a_{3}\right)\right\}\right.$, c is a constant $\}$ is a region of attraction for all solutions initiating in the positive quadrant. 


\section{Proof:}

From model (3.1) we have,

$$
\begin{array}{ll}
\frac{d G}{d t}=-a_{1} G-a_{2} G I+a_{1} G_{b} ; & G(0)=I_{0} \\
\frac{d X}{d t}=-a_{2} X+a_{3} I-a_{3} I_{b} ; & X(0)=X_{0} \\
\frac{d I}{d t}=a_{4} G-a_{5} I+a_{5} I_{b} ; & I(0)=I_{0}
\end{array}
$$

Therefore, $\frac{d(G+X+I)}{d t}=-a_{1} G-a_{2} G I+a_{1} G_{b}-a_{2} X+a_{3} I-a_{3} I_{b}+a_{4} G-a_{5} I+a_{5} I_{b}$;

$$
\begin{aligned}
& \leq-\left(a_{1}-a_{4}\right) G-a_{2} X-\left(a_{5}-a_{3}\right) I+a_{1} G_{b}-a_{3} I_{b}+a_{5} I_{b} \\
& \leq-\left(a_{1}-a_{4}\right) G-a_{2} X-\left(a_{5}-a_{3}\right) I+M \\
& <-\min \left\{\left(a_{1}-a_{4}\right), a_{2},\left(a_{5}-a_{3}\right)\right\}(G+X+I)+M
\end{aligned}
$$

where, $M=a_{1} G_{b}-a_{3} I_{b}+a_{5} I_{b}$

Let $\delta=\min \left\{\left(a_{1}-a_{4}\right), a_{2},\left(a_{5}-a_{3}\right)\right\}$

Thus $G+X+I<c e^{-\delta t}+\frac{M}{\delta}$.

\subsection{Disease Free Equilibrium Points}

The disease free equilibrium of the above model (3.1) can be obtained by setting $\frac{d G}{d t}=\frac{d X}{d t}=\frac{d I}{d t}=0$

Thus we have,

$-a_{1} G-a_{2} G I+a_{1} G_{b}=0$

$-a_{2} X+a_{3} I-a_{3} I_{b}=0$

$a_{4} G-a_{5} I+a_{5} I_{b}=0$

Since we have considered the disease free equilibrium $X=0, I=0$. Thus the above system (3.1) reduces to,

$-a_{1} G_{0}+a_{1} G_{b}=0$

$G_{0}=\frac{a_{1} G_{b}}{a_{1}}$

$\therefore G_{0}=G_{b}$

Thus, the disease free equilibrium point is $E_{0}=\left(G_{b}, 0,0\right)$.

Again for the endemic equilibrium point $E_{\text {end }}$, we find $E_{\text {end }}=\left(G^{*}, X^{*}, I^{*}\right)$

where, $G^{*}=\frac{-\left(a_{1} a_{5}+a_{2} a_{5}\right) \pm \sqrt{\left(a_{1} a_{5}+a_{2} a_{5}\right)^{2}-4 a_{1} a_{2} a_{4} a_{5} G_{b}}}{2 a_{2} a_{4}}, X^{*}=\frac{a_{3}}{a_{2}}\left(I^{*}-I_{b}\right), I^{*}=\frac{a_{1}}{a_{5}} G^{*}+I_{b}$ 


\subsection{Linearization of the Model}

Consider the Jacobian matrix of (3.1) given by

$J=\left(\begin{array}{ccc}-a_{1}-a_{2} I & 0 & -a_{2} G \\ 0 & -a_{2} & a_{3} \\ a_{4} & 0 & -a_{5}\end{array}\right)$

At $\left(G^{*}, X^{*}, I^{*}\right)$

$J^{*}=\left(\begin{array}{ccc}-a_{1}-a_{2} I^{*} & 0 & -a_{2} G^{*} \\ 0 & -a_{2} & a_{3} \\ a_{4} & 0 & -a_{5}\end{array}\right)$

We now use the transformation $G=g+G, X=x+X^{*}, I=i+I^{*}$ and then linearize the system

$\left(\begin{array}{c}\dot{g} \\ \dot{x} \\ \dot{i}\end{array}\right)=J^{*}\left(\begin{array}{c}g \\ x \\ i\end{array}\right)=\left(\begin{array}{ccc}-a_{1}-a_{2} I^{*} & 0 & -a_{2} G^{*} \\ 0 & -a_{2} & a_{3} \\ a_{4} & 0 & -a_{5}\end{array}\right)\left(\begin{array}{c}g \\ x \\ i\end{array}\right)$

We get the linearized system as

$\dot{g}=-a_{1} g-a_{5} I^{*} g-a_{2} G^{*} i$

$\dot{x}=-a_{2} x+a_{3} i$

$i=a_{4} g-a_{5} i$

4.4 Stability Analysis

Theorem 4.1: The interior-equilibrium point $\left(G^{*}, X^{*}, I^{*}\right)$ is locally asymptotically stable if $\left(a_{4}-a_{2} G^{*}\right)^{2}<8 a_{2}\left(a_{1}+a_{2} I^{*}\right)$.

\section{Proof:}

Consider the Lyapunov function

$L=\frac{1}{2}\left(g^{2}+x^{2}+i^{2}\right)$

Hence

$\dot{L}=\frac{1}{2} 2 g \dot{g}+\frac{1}{2} 2 x \dot{x}+\frac{1}{2} 2 i \dot{i}$ 
$\dot{L}=\left(-a_{1}-a_{2} I^{*}\right) g^{2}+\left(-a_{2} x+a_{3} i\right) x-a_{2} G^{*} i g+\left(a_{4} g-a_{5} i\right) i$

$\dot{L}=-\left(a_{1}+a_{2} I^{*}\right) g^{2}+\left(a_{1}-a_{2} G^{*}\right) i g-a_{2} x^{2}+a_{3} i x-a_{5} i^{2}$

$\dot{L}=-\frac{1}{2} p g^{2}+q i g-\frac{1}{2} r i^{2}-\frac{1}{2} s x^{2}+t i x$

where $p=2\left(a_{1}+a_{2} I^{*}\right), q=\left(a_{1}-a_{2} G^{*}\right), r=2 a_{5}, s=2 a_{2}, t=a_{3}$

The sufficient condition for $\dot{L}$ to be negative definite is that

$q^{2}<p r s$

i.e., $\left(a_{4}-a_{2} G^{*}\right)^{2}<8 a_{2} a_{5}\left(a_{1}+a_{2} I^{*}\right)$

which is the condition that the parameters must satisfy so that the critical point $\left(G^{*}, X^{*}, I^{*}\right)$ is locally asymptotically stable.

\section{Numerical Simulations}

We have discussed the locally asymptotically stability inside the body of normal person as well as different types of diabetic patient at the endemic equilibrium point $E_{\text {end }}$ who are suffering from diabetes mellitus but the results from each patient is different and it is explained with the help of graphical illustrations (see in Figures 5.1-5.4) and different types of parameters values. In this simulation we take one normal person and three diabetes patients.
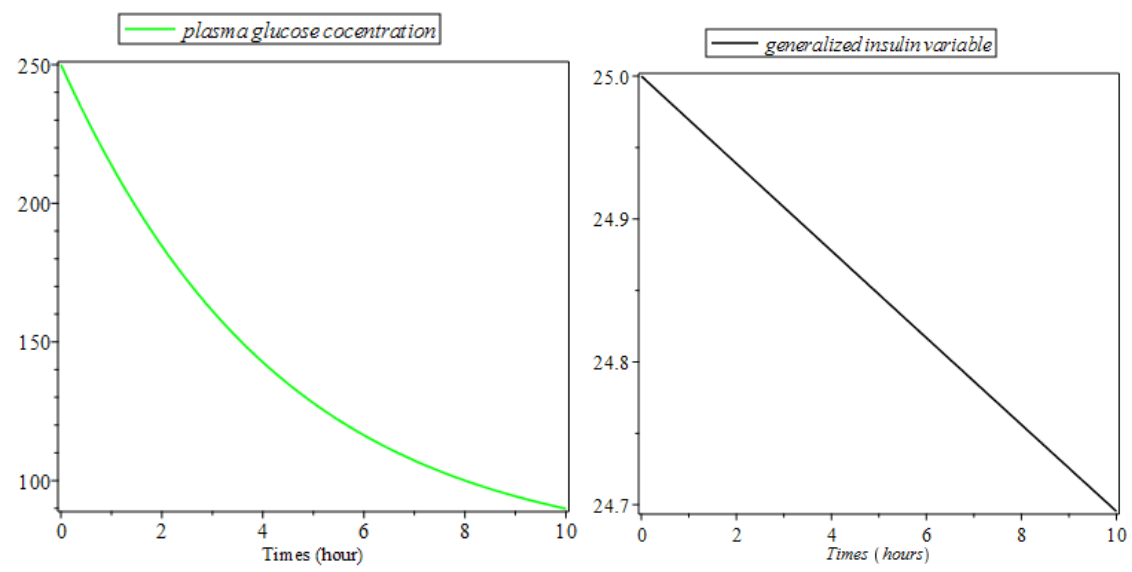


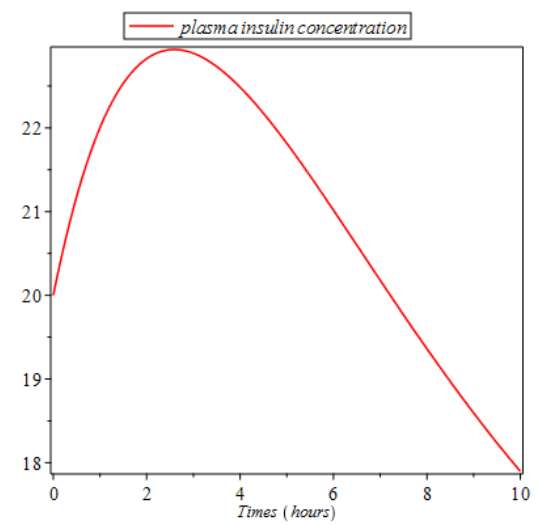

Figure 5.1: Glucose-insulin regulatory system for normal person, with different parameters $a_{1}=0.2, a_{2}=.00123$, $a_{3}=6.92 \times 10^{-6}, a_{4}=0.0249, a_{5}=-.2659, G_{b}=80, I_{b}=7$.
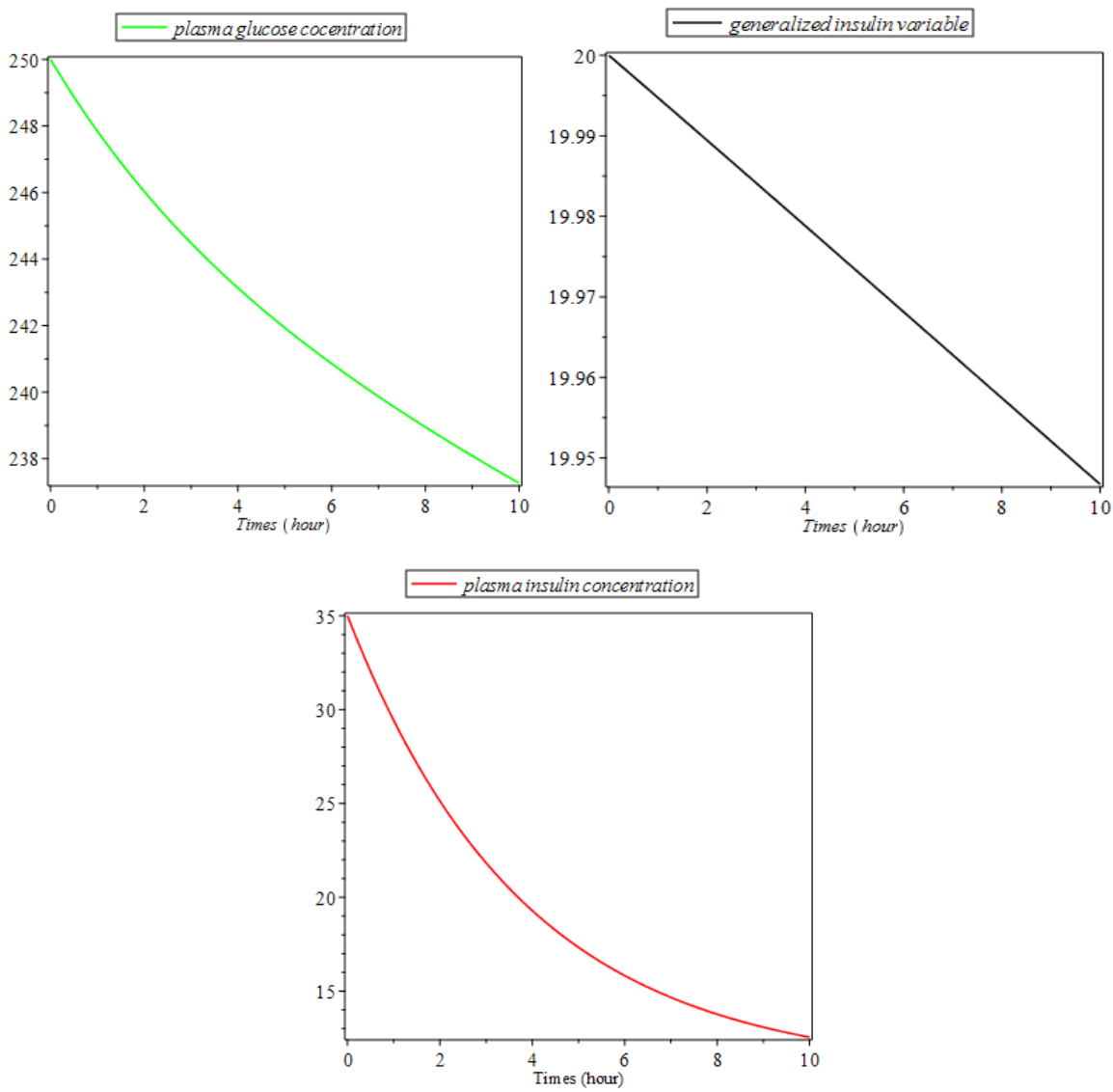

Figure 5.2: Glucose-insulin regulatory system for diabetes patient 1 , with different parameters $a_{1}=0, a_{2}=0.0027, a_{3}=5.3 \times 10^{-6}, a_{4}=0.0042, a_{5}=.264, G_{b}=80, I_{b}=7$. 

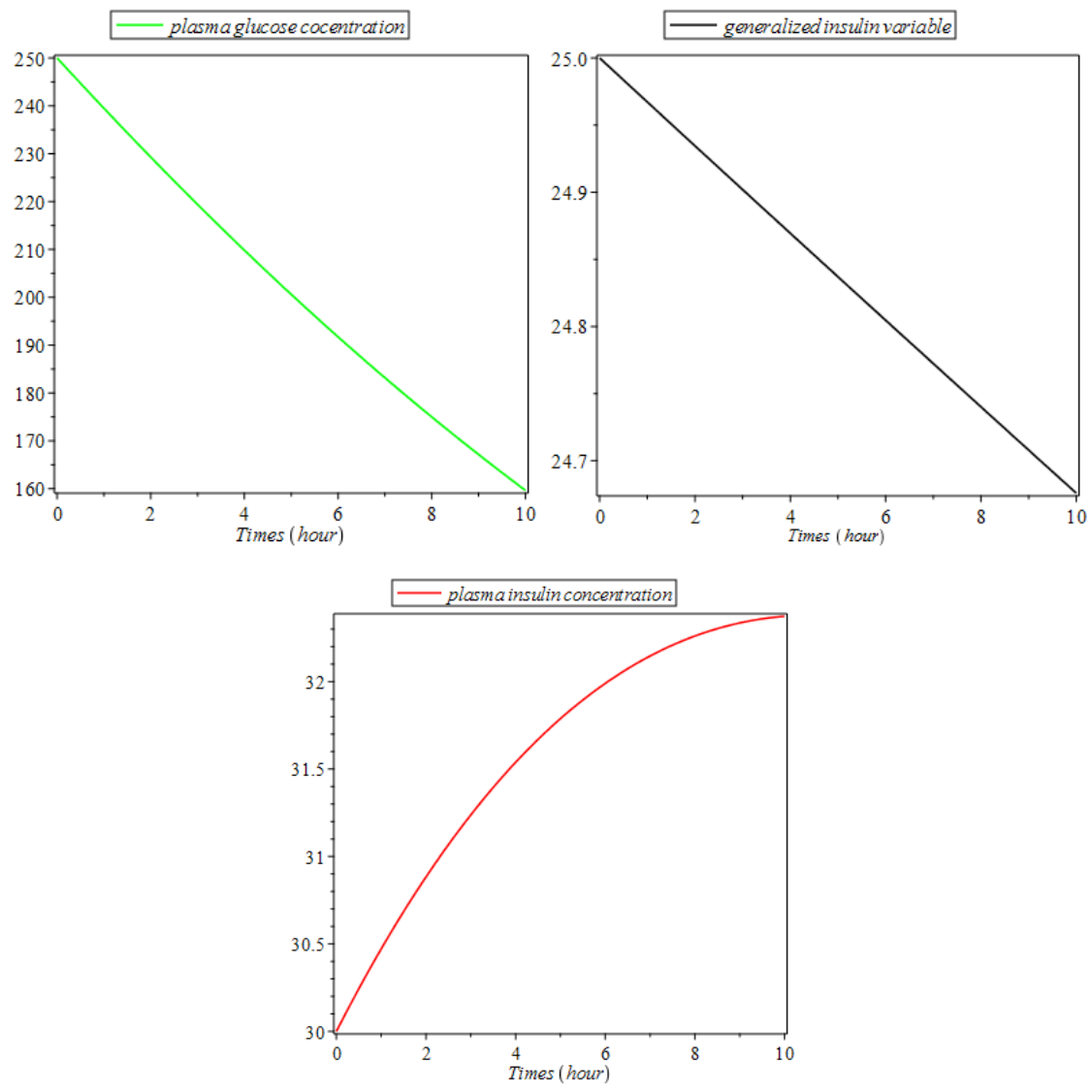

Figure 5.3: Glucose-insulin regulatory system for diabetes patient 2, with different parameters $a_{1}=0, a_{2}=0.00142, a_{3}=115.94 \times 10^{-6}, a_{4}=0.0046, a_{5}=0.2814, G_{b}=80, I_{b}=7$.
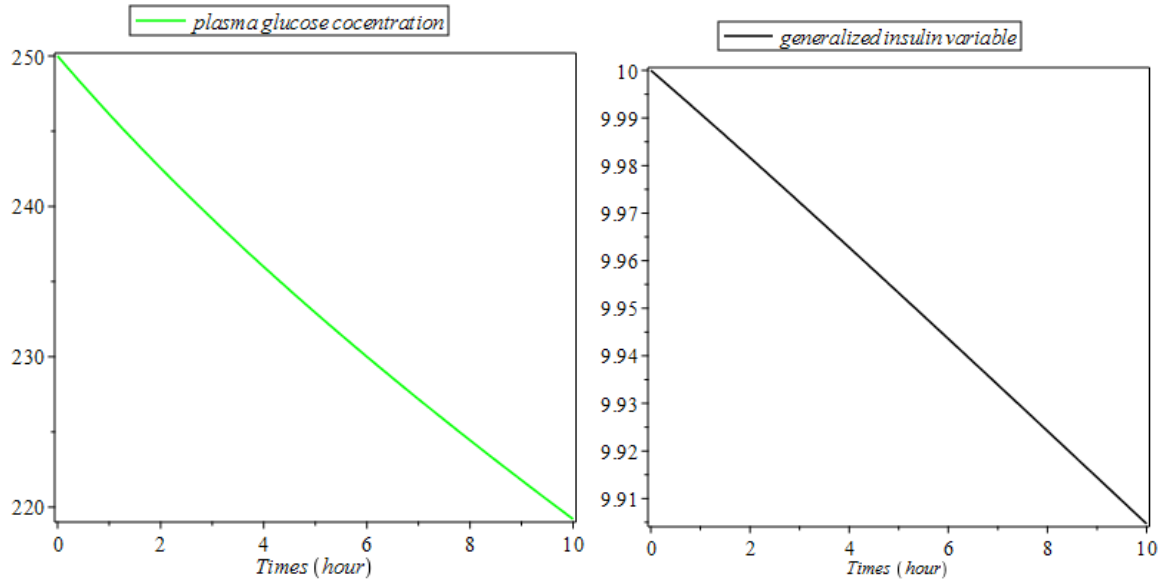


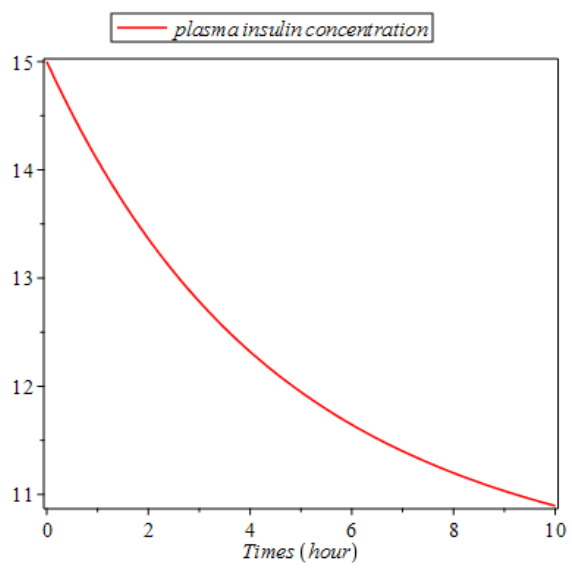

Figure 5.4: Glucose-insulin regulatory system diabetes patient 3, with different parameters $a_{1}=0, a_{2}=0.001072, a_{3}=216 \times 10^{-6}, a_{4}=0.0038, a_{5}=0.2465, G_{b}=80, I_{b}=7$.

From Figure 5.1, we observe that the $E_{\text {end }}$ is stable in the presence of different parameters. In this Figure we have shown that when glucose and insulin is given to the normal persons the glucose concentration level and insulin concentration level become very high and as time passes the level become stable. The same can be seen in case of generalized insulin variable there is no change even after some time it will remain same and this represent the glucose insulin regulation in the normal human body. In Figure 5.2, it is observed that $E_{\text {end }}$ is unstable because in that case the glucose level is very high from the basal level also the insulin level is decreased from the basal level of insulin in presence of different parameter values and the similar finding is shown in Figure 5.4. Also, in Figure 5.3, we have shown that in presence of different parameter values the glucose and insulin level become very high and this is the complex situation of diabetes patients.

\section{Conclusions}

Now a days, diabetes is the top at the list of non-infectious diseases in Bangladesh. The prevalence of diabetes rate is increasing day by day and becoming a great threat to us. In this paper, we have shown a brief report of diabetes mellitus of Bangladesh from 1980 to 2014. Again, we have discussed the mathematical presentation of glucose-insulin regulation inside the human body in a three-compartmental model. In this model, we take plasma glucose concentration $G(t)$, generalized insulin variable $X(t)$ for the remote compartment and plasma insulin concentration $I(t)$. In this study, we use a nonlinear system whose validity is proved by positivity test supported by simulations results. At disease free equilibrium point, the model is assumed to be stable and later we conclude the stable condition for the endemic equilibrium points. The numerical solutions have been carried out to show the variability of Glucose, insulin and plasma glucose concentration for different kind of diabetic patients as well as normal a patient at different equilibrium points and according to the Figures 5.1-5.4, we observe that how a diabetic patient controls his glucose and insulin level using different parameter values because control of glucose insulin level is so much important for the diabetes patients. 


\section{ACKNOWLEDGEMENTS}

The authors would like to thank the anonymous reviewers for their valuable suggestions and fruitful comments which help to incorporate considerable improvement in the revised manuscript.

\section{REFERENCES}

[1] Ackerman, E., Gatewood, L.C., Rosevear, J. W., and Molnar, G. D., (1965). Model studies of blood glucose regulation. Bull. Math. Biophys, 27: 21-27.

[2] Bortolon, L. N. M., de P, L., Triz. L., de S, B., de Sa, Faustino, L. B. C., Rocha, D. R. T. W. and Arbex, A. K., (2016). Gestational Diabetes Mellitus: New Diagnostic criteria. Open Journal of Endocrine and Metabolic Diseases, 6, 13-19.

[3] Biswas, M. H. A., (2013). Necessary Conditions for Optimal Control Problems with State Constraints: Theory and Applications. PhD Thesis, University of Porto, Porto.

[4] Biswas, M. H. A., (2012). Model and Control Strategy of the Deadly Nipah Virus (NiV) Infections in Bangladesh. Research \& Reviews in Biosciences, 6, 370-377.

[5] Bergman, R. N. and Cobelli, C., (1980). Minimal modeling partition analysis and the estimation of insulin sensitivity. Federation Proceedings, 39, 110-115.

[6] Bertram, R. and Pernarowski, M., (1998). Glucose Diffusion in Pancreatic Islets of Langerhans. Biophysical J., 74, 1722-173.

[7] Biswas, M. H. A., (2014). On the Evolution of AIDS/HIV Treatment: An Optimal Control Approach. Current HIV Research, 12, 1-12.

[8] Bocharov, G. A. and Rihan, F. A., (2000). Numerical modelling in biosciences using delay differential equations, J. Comput. Appl. Math., 125, 183-199.

[9] Cerasi, E., Khanina, G. and Drozdov, M., (1974). A mathematical model for the glucose induced insulin release in man, Eur. J. Clin. Invest, 4, 276-278.

[10] Definition, Diagnosis and Classification of Diabetes Mellitus and its Complications. Part 1: Diagnosis and Classification of Diabetes Mellitus. Geneva, World Health Organization, (2015).

[11] Grodsky, G. M., (1972). A threshold distribution hypothesis for packet storage of insulin and its mathematical modeling, The J. Clin. Invest., 51, 2047-2059.

[12] International Diabetes Federation: IFD report, (2016). [http://www.idf.org/home/index.cfm]

[13] Li, J., Kuang, Y. and Mason, C., (2006). Modeling the glucose-insulin regulatory system and ultradian insulin secretory oscillations with two explicit time delays. Journal of Theoretical Biology, 242, 722735.

[14] Liu, W. and Tang, F., (2008). Modeling a simplified regulatory system of blood glucose at molecular levels. Journal of Theoretical Biology, 252, 608-620.

[15] Mondal, M. K., Hanif, M. and Biswas, M. H. A., (2017). A mathematical analysis for controlling the spread of Nipah virus infection. International journal of modeling and simulation, 37(3):185-197.

[16] Pacini, G. and Bergman, R. N., (1996). MINMOD: A computer program to calculate insulin sensitivity and pancreatic responsibility from the frequently sampled intravenous glucose tolerance test. Computer Methods and Programs in Biomedicine, 23, 113-122.

[17] The world health report, Today's challenges. Geneva, World Health Organization, 2016. [http://www.who.int/whr/2016/en]

[18] Tarın, C., Teufel, E., Pico, J., Bondia, J. and Pfleiderer, H. J., (2005). Comprehensive pharmacokinetic model of insulin glargine and other insulin formulations, IEEE Trans. Biomed. Eng., 52 (12), 19942005.

[19] Wang, H., Li, J. and Kuang, Y., (2009). Enhanced modelling of the glucose-insulin system and its applications in insulin therapies, J. of Biological Systems, 3, 22-38. 